\title{
Mode spectra of magnetic and dielectric plasmon spheres obtained from Mie scattering and free oscillation theories
}

\author{
Jerzy Krupka ${ }^{1}$, Bartlomiej Salski ${ }^{2, a} \mathbb{D}$, Adam Pacewicz ${ }^{2}$, and Pawel Kopyt ${ }^{2}$ \\ 1 Institute of Microelectronics and Optoelectronics, Warsaw University of Technology, Warsaw, Poland \\ 2 Institute of Radioelectronics and Multimedia Technology, Warsaw University of Technology, Warsaw, Poland
}

Received 27 May 2021 / Accepted 22 November 2021 / Published online 31 December 2021

(C) The Author(s) 2021

\begin{abstract}
This paper presents Mie scattering theory as compared to rigorous electromagnetic theory of free oscillations in magnetic and electric plasmon spheres. It is shown that the maxima of Mie scattering and absorption spectra well correspond to resonance frequencies of plasmon modes occurring in dielectric and magnetic spheres, similarly as it takes place for ordinary dielectric resonator modes. Mie theory is well applicable to determine resonance frequencies and scattering parameters of spherical plasmons. However, this theory cannot be applied to determine intrinsic properties of modes induced in the object by the incident plane wave, like quality factors. On the contrary, rigorous electromagnetic theory of free oscillations allows one to determine the complex resonance frequency of each mode that can occur in a given object, and the corresponding quality factor accounting for various kind of losses, including medium and radiation losses. The advantage of the free oscillations theory, as shown in this paper, is in the determination of the quality factors of modes occurring in magnetic plasmon spheres made of a strongly dispersive magnetic medium.
\end{abstract}

\section{Introduction}

Scattering of electromagnetic (EM) waves on dielectric objects is a well-recognized physical phenomenon. A rigorous theory of scattering on spherical-shaped objects was first described in [1] and subsequently addressed in hundreds of papers and several books [26 . The relationship between Mie scattering ripples and free oscillation resonances has been already rigorously explained by several authors, e.g., [7-13]. The scattering problem has an analytical solution in the form of an infinite series for ellipsoidal samples made of isotropic and uniform media. Depending on the diameter of the spherical sample, $d$, relative to the freespace wavelength of the incident plane wave, $\lambda$, three kinds of scattering are distinguished: Rayleigh scattering $(d<<\lambda)$, Mie scattering $(d \sim \lambda)$, and geometric scattering $(d>>\lambda)$. Mie scattering theory (Mie) is sometimes referred to as resonance scattering, because both scattered and absorbed waves create discrete spectra due to modes excited in the sphere. In fact, spherical samples are EM resonators whose free oscillations (FO) are characterized by an infinite series of complex resonance frequencies. The boundary value problem for Maxwell's curl equations has analytical solutions for isotropic spheres, which allows determining the resonance frequencies and Q-factors (i.e., losses) of the modes occurring in the object.

\footnotetext{
a e-mail: bartlomiej.salski@pw.edu.pl (corresponding author)
}

Although those two methods deal with the sphere in a completely different manner, as Mie is a deterministic problem and the boundary value problem solves for FO, very good correlations between resonance frequencies of a dielectric sphere and singularities in Mie absorption spectra occur. The main goal of this paper is to compare resonance frequencies obtained with the two methods for plasmonic modes. Newly recognized magnetic plasmon resonances (MPRs) occurring in ferrimagnetic spheres will be especially investigated in this paper. According to the authors' knowledge, such a comparative study of the two theories for magnetic plasmon modes has never been undertaken before. For historical reasons, comparative computations are also performed for ordinary resonances occurring in dielectric spheres with focus on their quality factors due to radiation losses.

\section{Theory}

\subsection{Ordinary dielectric spherical resonators}

All computations of scattering efficiency presented in this paper have been performed employing functions developed in $[3,4]$. Analytical solutions of the eigenvalue problem for Maxwell's curl equations in spherical dielectric resonators have been presented in [14] for homogeneous dielectrics, in [15] for multilayered dielectrics, and in [16-19] for magneto-dielectric materials (e.g., 
(a)

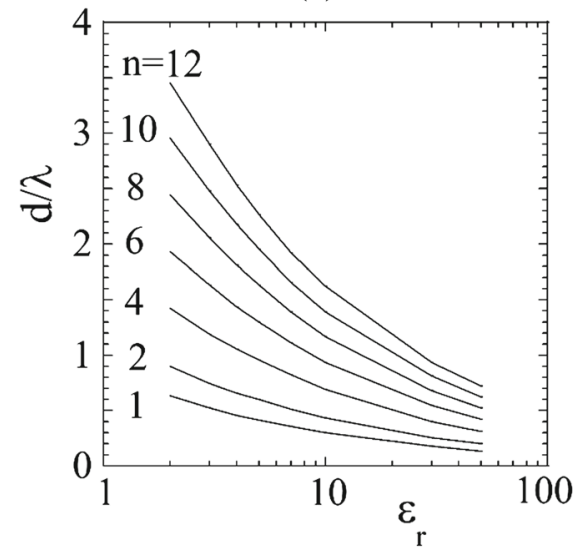

(b)

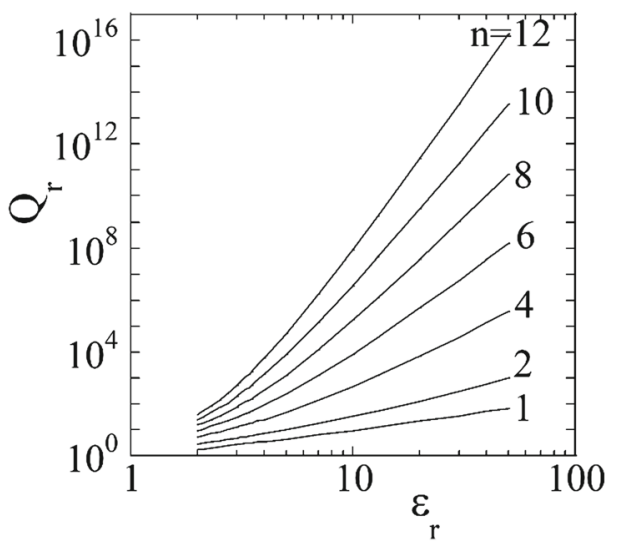

Fig. 1 a Normalized eigen-frequencies $(d / \lambda)$ of $\mathrm{TE}_{n 01}$ modes for a spherical lossless dielectric sample located in vacuum versus its relative permittivity for $\mu_{r}=1$, and the corresponding $\mathbf{b} Q$-factors due to radiation $\left(Q_{r}\right)$

ferrites). In principle, the solution of the eigenvalue problem for $\mathrm{FO}$ of a resonance system can be expressed in the form of complex eigen-frequencies, $\hat{\omega}_{s}=\omega_{s}^{\prime}+j \omega_{s}^{\prime \prime}$, corresponding to specific eigen-functions (modes), with $Q$-factors accounting for losses that can be evaluated as $Q_{s}=\frac{\omega_{s}^{\prime}}{2 \omega_{s}^{\prime \prime}}$. For the isotropic homogeneous magnetodielectric sphere of diameter $d$, the eigenvalue problem is reduced to two separate transcendental equations (TDE) for $\mathrm{TE}_{n m p}$ and $\mathrm{TM}_{n m p}$ mode families [see (B.1) and (B.2) in Appendix B].

Ordinary EM resonances appear when both permittivity and permeability are positive, whereas plasmon type resonances appear when one of the two quantities is negative. Recently, it has been shown that resonances observed and employed in YIG (yttrium iron garnet) filters and oscillators are, in fact, MPRs for circularly polarized (CP) modes [16]. The effective permeability tensor corresponding to that mode can be considered isotropic and dispersive in a plane transverse to the static magnetic field bias. Formulae for the effective permeability corresponding to the clockwise CP MPR are given in Appendix A. More theoretical details about MPRs can be found in [16-19].

Results of computations of normalized resonance frequencies and $Q$-factors due to radiation for $\mathrm{TE}_{n 01}$ modes occurring in spherical dielectric samples located in vacuum as a function of its relative permittivity are shown in Fig. 1. As it is seen in Fig. 1b, the $Q$-factors due to radiation increase by several orders of magnitude with the increasing elevation mode index, $n$, as the mode becomes more and more confined within the sphere, which in turn suppress radiation losses. The tendency is enhanced in dielectric spheres with high permittivity. For low-permittivity samples, radiation losses are relatively large and the corresponding $Q$-factors due to radiation are small. Commercially available cylindrical dielectric resonators with permittivity in the range 20-40 operate on a low-order mode and have to be situated in a metal shield to avoid radiation losses.
Subsequently, Mie extinction coefficients, $Q_{\text {ext }}$, which account for both scattering and absorption, were computed using functions available in [4] for $\varepsilon_{r}=4$ and $\varepsilon_{r}=10$ and the results are shown in Fig. 2 and in Table 1. Maxima of $Q_{\text {ext }}$ correspond well to the eigen-frequencies of dielectric resonator modes computed with the relevant TDE. Agreement between spectral locations of $Q_{\text {ext }}$ maxima and computed eigenfrequencies are to within 4-5 significant digits (see Table 1), except for the first two modes which are highly radiative, as it is seen in Fig. 1b, so their resonance frequencies are slightly deviated due to losses.

\subsection{Magnetic and electric plasmon resonators}

In a similar way as for ordinary resonances in nondispersive dielectric spheres, solutions of the TDE [i.e., $d / \lambda$ values being the solutions of (B.1) or (B.2)Appendix B] can be compared with $\mu_{r}$ or $\varepsilon_{r}$ corresponding to the maxima of $Q_{\text {ext }}$ obtained in plasmonic regime, i.e., for $\mu_{r}<0$ or for $\varepsilon_{r}<0$. According to the magneto-static and the electro-static models, plasmonic resonances for a small spherical magnetic (dielectric) sample situated in vacuum appear if the relative permeability (permittivity) of the sample is equal to $\mu_{r}=-\frac{n+1}{n},\left(\varepsilon_{r}=-\frac{n+1}{n}\right)$, where $n=1,2,3, \ldots$ Electrodynamic theory of plasmon resonances in spheres agrees with the electro- and magneto-static theories only in the Rayleigh scattering regime when $d<<\lambda$. Electrodynamic theory of electric plasmon resonances (EPRs) is well established (e.g., for noble metal spheres at optical frequencies), while theory of MPRs in gyromagnetic spheres has only been recently developed [1619]. It has been shown that MPRs correspond to the $\mathrm{TE}_{n 01}$ modes of an isotropic spherical sample having an effective permeability as in (A.3) (see Appendix A). It should be underlined that the effective permeability of such a material is highly dispersive (i.e., frequencydependent).

For a given radius of the sample, its permittivity, internal static magnetic field, saturation magnetiza- 
(a)

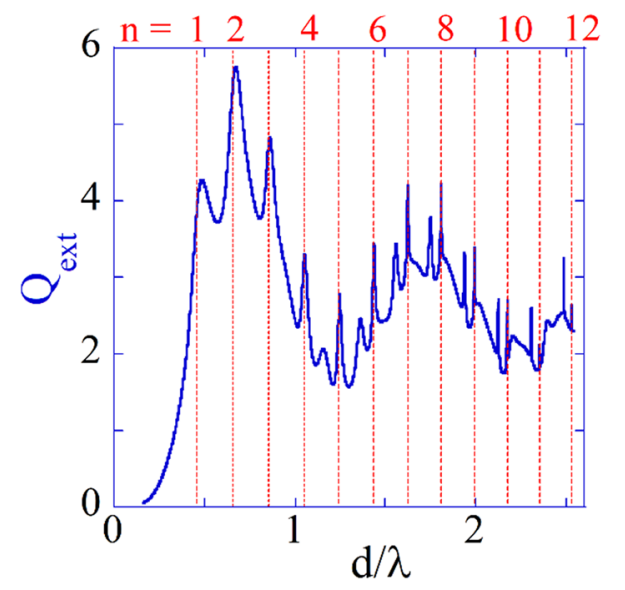

(b)

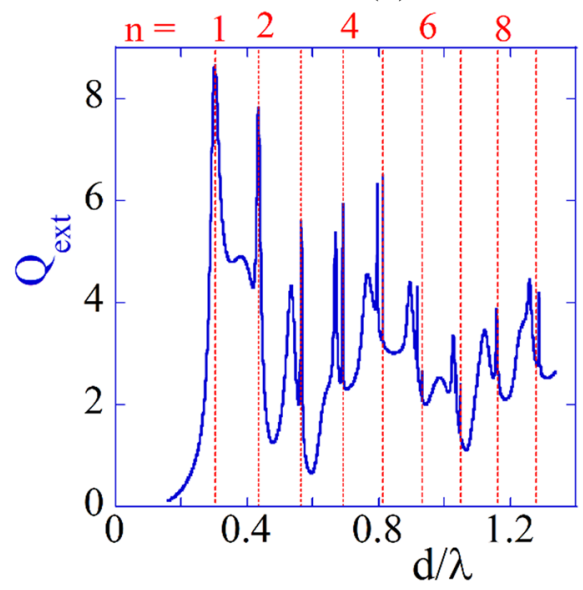

Fig. 2 Spectra of $Q_{\text {ext }}$ computed with Mie theory for $\mathbf{a} \varepsilon_{r}=4$ and $\mathbf{b} \varepsilon_{r}=10$. Vertical dashed lines correspond to the normalized eigen-frequencies $(d / \lambda)$ of $\mathrm{TE}_{n 01}$ modes computed with the TDE as in (B.1) assuming $\mu_{r}=1$

Table 1 Normalized frequency $(d / \lambda)$ for $\mathrm{TE}_{n 01}$ and $\mathrm{TM}_{n 01}$ modes computed with TDE and Mie theory for the isotropic dielectric sphere $\left(\varepsilon_{r}=10, \mu_{r}=1\right)$

\begin{tabular}{lllllllll}
\hline Mode family & Method & $n=1$ & $n=2$ & $n=3$ & $n=4$ & $n=5$ \\
\hline $\mathrm{TE}_{n 01}$ & TDE Eq. (B.1) & 0.29915 & 0.43482 & 0.56519 & 0.69076 & 0.81258 \\
& Mie & 0.30219 & 0.43496 & 0.56525 & 0.69072 & 0.81257 \\
$\mathrm{TM}_{n 01}$ & TDE Eq. (B.2) & 0.43200 & 0.53661 & 0.66953 & 0.79644 & 0.91838 \\
& Mie & 0.38177 & 0.53674 & 0.66933 & 0.79642 & 0.91837 & 1.03715 \\
& & &
\end{tabular}

tion, and Gilbert damping factor (or ferromagnetic linewidth, $\Delta H$ ), it is possible to obtain solutions of the TDE, as in (B.1), being the resonance frequencies of various $\mathrm{TE}_{n 0 p}$ modes. In addition, the frequencydependent effective permeability values at these frequencies are obtained. The lowest frequency $\mathrm{TE}_{101}$ mode corresponds to the so-called mode of uniform precession (following simplified magneto-static theory of resonances [20]) for which $\mu_{r}=-2$ (for the lossless case). This mode is commonly employed in YIG filters and oscillators. One can easily derive from (A.4) that for this mode $\mathrm{w}-H_{0 r}=\frac{1}{1-\mu_{r}}=1 / 3$, which shows that the normalized resonance frequency, $w$, linearly depends on the normalized static magnetic field, $H_{0 r}$, as long as $d<<\lambda$ (see definitions in the Appendix A). Numerical solutions of TDE, as in (B.1), for larger $d / \lambda$ are shown in Fig. 3. It can be noted that as $d / \lambda$ increases, $\mathrm{w}-H_{0 r}$ values converge to 0 and the effective $\mu_{r}$ converges to $-\infty$ (which is the ferromagnetic resonance condition in an unbounded medium).

It should be noted that the approximate Mie scattering results $[21,22]$ well agree with the TDE solutions for small $d / \lambda$. These days more accurate Mie scattering computer programs are available (more terms in the EM field expansion series), so the agreement between FO and Mie results takes place in a broad range of $d / \lambda$ values. Comparisons of Mie results based on Mie scattering software and TDE solutions are not straightforward. For a given $H_{0 r}$ column vector, the TDE pro-

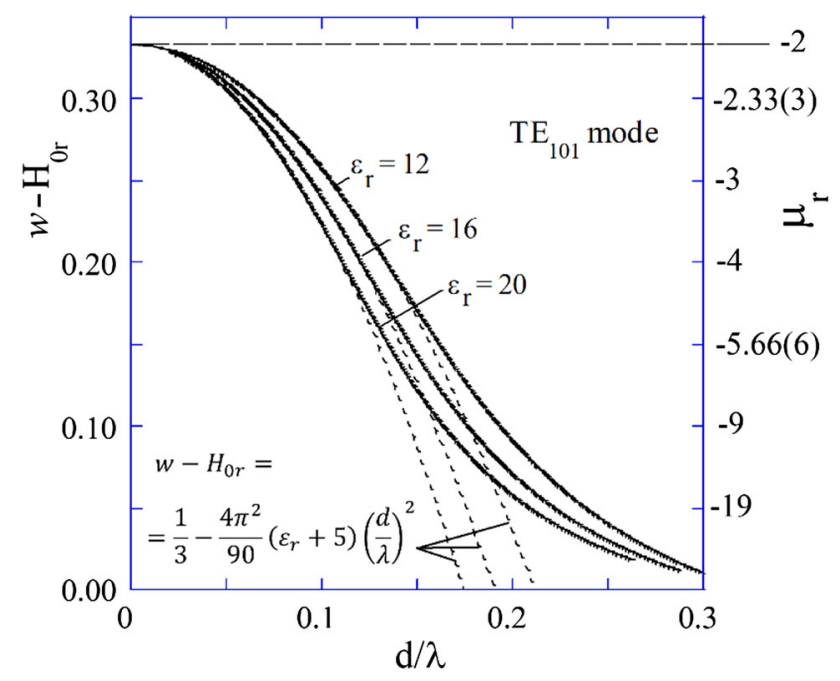

Fig. 3 Results of $w-H_{0 r}$ and the effective permeability computations versus the normalized frequency $(d / \lambda$ obtained from TDE, as in (B-1). Dashed lines and the formula in the lower left corner correspond to the approximate Mie scattering solution $[21,22]$

duces a two-column matrix. The first column contains normalized frequency $d / \lambda$ values and the second column contains the effective permeability values for these frequencies. Mie scattering software returns the extinction coefficients vector $Q_{\text {ext }}$ for given $d / \lambda$ vector and 
(a)

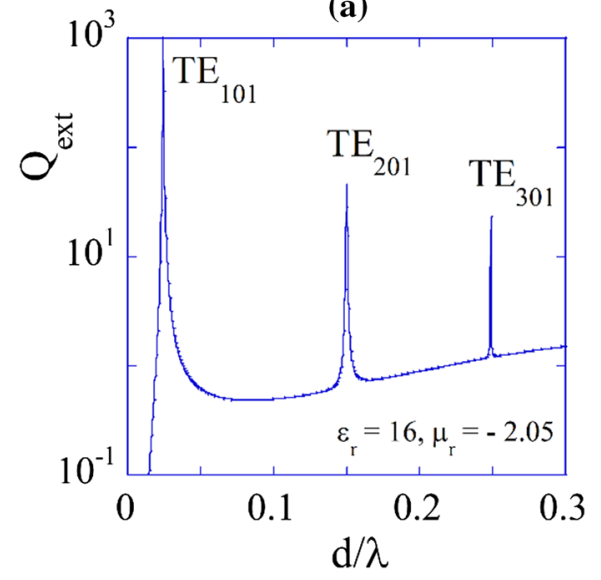

(b)

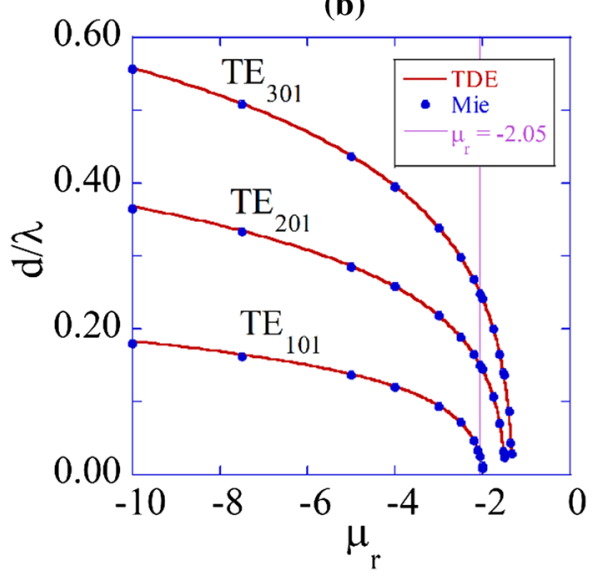

Fig. 4 a Computed spectrum of extinction coefficient $Q_{\text {ext }}$ as function of $d / \lambda$ for $\varepsilon_{r}=16$ and $\mu_{r}=-2.05 e$. b Normalized resonance frequencies $(d / \lambda)$ of three MPRs as functions of $\mu_{r}$ in a spherical sample having $\varepsilon_{r}=16$. Solid lines correspond to TDE solutions, while dots to results obtained as $d / \lambda$ values corresponding to maxima of $Q_{\text {ext }}$ in Mie theory

Table 2 Normalized frequency $(d / \lambda)$ versus the effective permeability $\mu_{r}$ for the TE $\mathrm{E}_{101}$ mode computed with TDE and Mie theories for the isotropic magneto-dielectric sphere $\left(\varepsilon_{r}=16\right)$

\begin{tabular}{llllllllll}
\hline$\mu_{r}$ & -10 & -7.5 & -5 & -4 & -3 & -2.5 & -2.2 & -2.1 & -2.05 \\
\hline TDE & 0.18323 & 0.16501 & 0.13766 & 0.12082 & 0.09433 & 0.07132 & 0.04735 & 0.03409 & 0.02431 \\
Mie & 0.18323 & 0.16499 & 0.13767 & 0.12081 & 0.09434 & 0.07132 & 0.04734 & 0.03408 & 0.02432 \\
\hline
\end{tabular}

fixed values of $\varepsilon_{r}$ and $\mu_{r}$. Mie computations of the plasmonic resonance frequencies require two steps. In the first step, $Q_{\text {ext }}$ is computed for an assumed $d / \lambda$ vector and fixed values of $\varepsilon_{r}$ and $\mu_{r}\left(\mu_{r}\right.$ is taken from the TDE computations). In the second step, the normalized resonance frequencies $d / \lambda$ are obtained as values corresponding to the $Q_{\text {ext }}$ maxima. Figure 4 a shows computation results of $Q_{\text {ext }}$ versus $d / \lambda$ for $\varepsilon_{r}=16$ and $\mu_{r}=-2.05 e$. Normalized resonance frequencies, $d / \lambda$, presented in Fig. $4 \mathrm{~b}$ were obtained as the $d / \lambda$ values corresponding to the maxima of several $Q_{\text {ext }}$ plots (for different $\mu_{r}$ ) and are listed in Table 2 for the $\mathrm{TE}_{101}$ mode. As it is seen in Table 2, agreement between Mie and FO (TDE) results is excellent to a relative accuracy better than $0.1 \%$.

In Fig. 5a, computation results of $Q_{\text {ext }}$ versus $d / \lambda$ for an electric plasmon sample having $\varepsilon_{r}=-2.05$ and $\mu_{r}=1$ are presented, whereas Fig. $5 \mathrm{~b}$ shows the dependence of the normalized resonance frequencies $(d / \lambda)$ versus effective permeability. Similarly, as for the MPRs, agreement between Mie and FO results for the EPRs is excellent. However, the spectrum of $Q_{\text {ext }}$ (see Fig. 5a) is different than for the MPRs (compare Fig. 4a). Moreover, normalized resonance frequencies $d / \lambda$ (see Fig. 5b) are different for $\varepsilon_{r}<0$ as compared to the corresponding negative values of $\mu_{r}$ (see Fig. 4b). These differences are related to the fact that the magnetic plasmon sample exhibits large permittivity $\left(\varepsilon_{r}=16\right.$ for YIG), whereas the electric plasmon sample is assumed to be non-magnetic $\left(\mu_{r}=1\right)$. Agreement between Mie and FO computations for the lowest order $\mathrm{TE}_{101}$ plasmon mode is much better than for the ordinary mode (compare results in Tables 1 and 2). This is related to much smaller radiation losses for plasmon modes due to the very small size of plasmonic samples. More details on this topic will be presented in the next section.

\subsection{Experiments and remarks on Q-factor computations}

Computations of $Q$-factors in resonators containing a dispersive medium with the effective permeability as in (A.2) can performed in two alternative ways. In the first approach, the complex frequency is used to extract the $Q$-factor, as it has been described in Sect.2.1. In the second approach, the frequency is real in all formulae. It should be also emphasized that due to dispersive properties of $\mu_{r}^{\prime}$, magnetic energy stored in the resonator is proportional to $\frac{\partial\left[\omega \mu_{r}^{\prime}(\omega)\right]}{\partial \omega}$ and not to $\mu_{r}^{\prime}$. The final expressions for the $Q$-factor related to losses in a magneto-dielectric medium with dispersive permeability and non-dispersive permittivity read

$$
Q_{\mathrm{md}}=\frac{\frac{1}{4}\left\{\iiint_{V} \varepsilon_{0} \varepsilon_{r}^{\prime}|\mathbf{E}|^{2} \mathrm{~d} v+\iiint_{V} \mu_{0} \frac{\partial\left[\omega \mu_{r}^{\prime}(\omega)\right]}{\partial \omega}|\mathbf{H}| \mathrm{d} v\right\}}{\frac{1}{2}\left(\iiint_{V} \varepsilon_{0} \varepsilon_{r}^{\prime \prime}|\mathbf{E}|^{2} \mathrm{~d} v+\iiint_{V} \mu_{0} \mu_{r}^{\prime \prime}(\omega)|\mathbf{H}|^{2} \mathrm{~d} v\right)}
$$


(a)

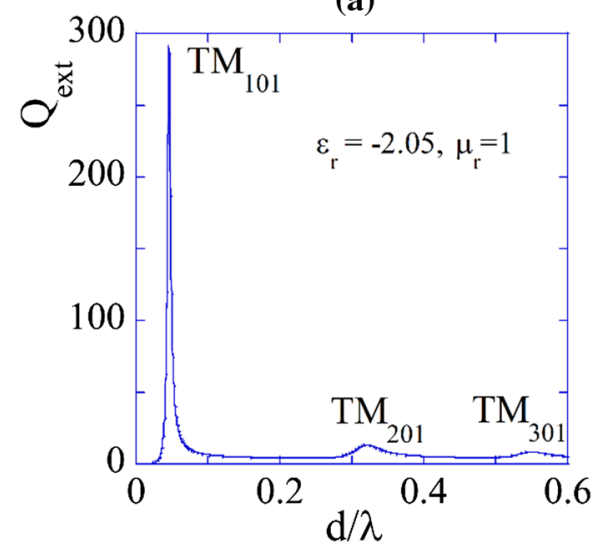

(b)

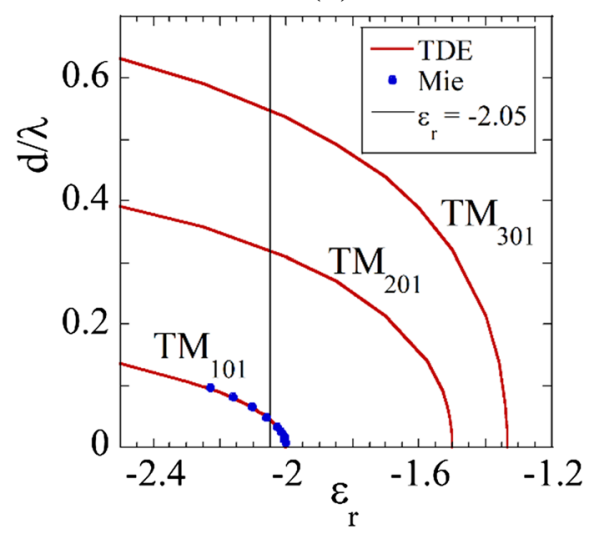

Fig. 5 a Computed spectrum of extinction coefficients $Q_{\text {ext }}$ obtained for $\varepsilon_{r}=-2.05 \mathrm{e}$ and $\mu_{r}=1.0$. b Normalized resonance frequencies $(d / \lambda)$ of three electric plasmon modes in a spherical sample. Solid lines correspond to TDE solutions, dots to results obtained as $d / \lambda$ values corresponding to maxima of $Q_{\text {ext }}$ in Mie theory

(a)

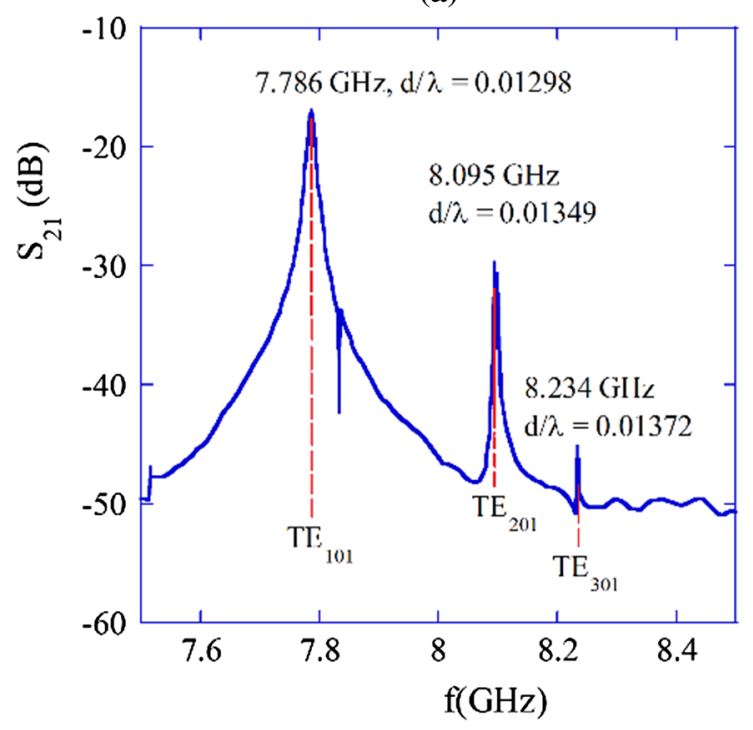

(b)

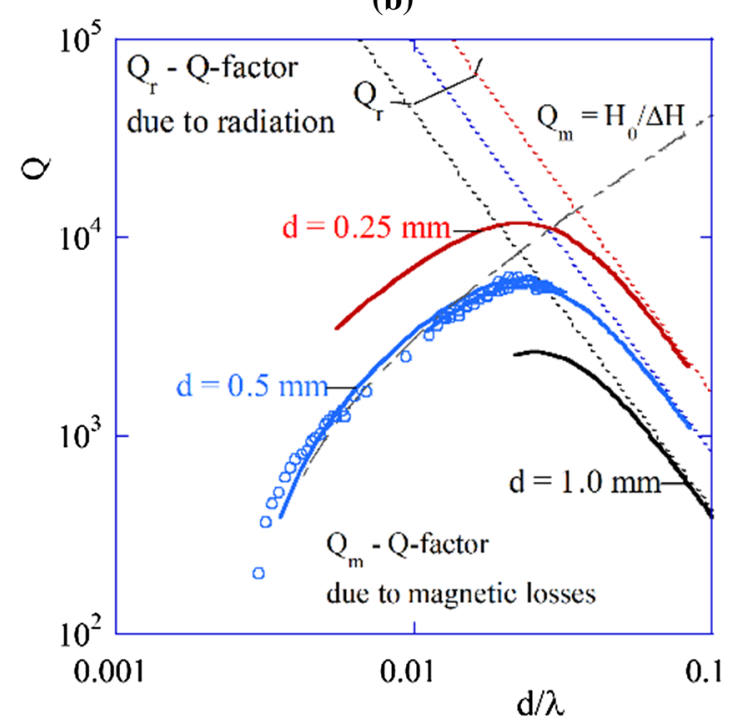

Fig. 6 a Measured transmission spectrum of a YIG sphere $\left(M_{\mathrm{S}}=140 \mathrm{kA} / \mathrm{m}, d=0.5 \mathrm{~mm}, \varepsilon_{r}=16\right)$ under a static magnetic bias $\left(H_{\text {ext }} \approx 217 \mathrm{kA} / \mathrm{m}\right)$ showing the first three $\mathrm{TE}_{n 01}$ magnetic plasmon modes. b Measured [18] (open circles) and computed with FO $Q$-factors of the $\mathrm{TE}_{101}$ mode for the YIG sphere versus normalized frequency

The denominator in (1) denotes the average total power dissipated in the resonator, $P_{\text {loss }}$, divided by $\omega$, whereas the numerator can be interpreted as the average total EM energy stored in the resonator. For the MPRs, the magnetic energy stored in the resonator per cycle can be even a few orders of magnitude larger than the average electric energy, especially for small samples. Agreement between these two approaches of $Q$-factor computations is excellent [19]. More information on this topic can be found in $[17,19]$.

Figure 6a shows measurement results of the transmission spectrum of a monocrystalline YIG sphere $\left(M_{\mathrm{S}}=\right.$ $\left.140 \mathrm{kA} / \mathrm{m}, d=0.5 \mathrm{~mm}, \varepsilon_{r}=16\right)$ under a static externally applied magnetic field bias $\left(H_{\text {ext }} \approx 217 \mathrm{kA} / \mathrm{m}\right)$. Details of the experimental setup have been presented in an earlier paper [23]. It is seen that highest peaks on the transmission spectrum correspond to the first three plasmon modes. From the measured resonance frequencies corresponding to these peaks, the saturation magnetization of the sample has been determined with a high precision [23].

Results of measurements, after [18], and FO computations of $Q$-factors for the YIG sphere are shown in Fig. $6 \mathrm{~b}$. The resonance frequency of the $\mathrm{TE}_{101}$ mode has been tuned by over a decade by varying the DC magnetic field bias. Computations of the total $Q$ factors have been performed assuming the ferromagnetic linewidth of the sample $\Delta H=0.5$ Oe (solid lines) and computations of the $Q$-factors due to radiation losses (broken lines) assuming $\Delta H=0$. Additionally, $Q$-factors due to magnetic losses, $Q_{\mathrm{m}}$, are presented for the sample having $d=0.5 \mathrm{~mm}$. It should be noted 
that $Q$-factors due to radiation losses for the plasmonic $\mathrm{TE}_{101}$ mode in a YIG sphere are few orders of magnitude larger than for the ordinary $\mathrm{TE}_{101}$ mode in a dielectric sample having the same permittivity as YIG (Fig. 1b). This is related to the size of the sample [18] which, for a given frequency, is several times smaller for the magnetic plasmon sample than for the dielectric one. This is confirmed by Mie computations where the peak of $Q_{\text {ext }}$ in Fig. 4a for the first plasmon mode is much sharper than the $Q_{\text {ext }}$ peak for the same mode in the dielectric sample (see Fig. 2b).

\section{Conclusions}

Mie and FO theories agree in the determination of the resonance frequencies for both plasmon and ordinary modes occurring in isotropic spheres. Agreement is up to 4-5 significant digits, except for highly radiative ordinary modes. Computations of the resonance frequencies with the aid of the Mie theory are typically faster than those based on the FO theory, and provide immediate results for scattering and absorption spectra of magneto/dielectric objects. However, FO theory allows one not only to accurately determine the resonance frequencies but also the $Q$-factors, especially for resonators containing dispersive media.

Acknowledgements This work was supported by the TEAM-TECH project entitled "High-precision techniques of millimeter and sub- $\mathrm{THz}$ band characterization of materials for microelectronics" operated within the Foundation for Polish Science TEAM TECH Programme co-financed by the European Regional Development Fund, Operational Programme Smart Growth 2014-2020. Partial support by the National Science Centre, project registration number 2018/31/B/ST7/04006 is also kindly acknowledged.

\section{Author contributions}

Jerzy Krupka contributed to the study of ordinary dielectric and plasmonic spheres with the aid of both TDE and Mie scattering theory. Bartlomiej Salski contributed to the study of magnetic plasmonic spheres with the aid of both TDE and Mie scattering theory. Adam Pacewicz contributed to the study of ordinary dielectric and plasmonic spheres with the aid of TDE. Pawel Kopyt contributed to the study of the Q-factor of plasmonic spheres with the aid of TDE.

Data availability statement This manuscript has no associated data or the data will not be deposited. [Authors' comment: Data is available upon request.]

Open Access This article is licensed under a Creative Commons Attribution 4.0 International License, which permits use, sharing, adaptation, distribution and reproduction in any medium or format, as long as you give appropriate credit to the original author(s) and the source, provide a link to the Creative Commons licence, and indicate if changes were made. The images or other third party material in this article are included in the article's Creative Commons licence, unless indicated otherwise in a credit line to the material. If material is not included in the article's Creative Commons licence and your intended use is not permitted by statutory regulation or exceeds the permitted use, you will need to obtain permission directly from the copyright holder. To view a copy of this licence, visit http://creativecomm ons.org/licenses/by/4.0/.

\section{Appendix A}

When a ferromagnetic medium is subjected to a uniform internal static magnetic field, $H_{0}$, directed along the $z$-axis of a cylindrical or Cartesian coordinate system, then its permeability tensor takes the following form [24,25]:

$$
\bar{\mu}=\mu_{0}\left[\begin{array}{ccc}
\mu & j \kappa & 0 \\
-j \kappa & \mu & 0 \\
0 & 0 & \mu_{z}
\end{array}\right] .
$$

For a spherical sample, the internal static magnetic field is related to the external (applied) static magnetic field $H_{\text {ext }}$ by the formula $H_{0}=H_{\text {ext }}-\frac{1}{3} M_{\mathrm{S}}-\delta H_{a}$, where $M_{\mathrm{S}}$ is saturation magnetization and $\delta H_{a}$ is a static magnetic field shift related to the crystal anisotropy.

For a high-frequency magnetic field which is orthogonal to $H_{0}$, and clockwise $\mathrm{CP}$ with respect to $H_{0}$, the permeability tensor, as in (A.1), is reduced to the diagonal form

$$
\bar{\mu}=\mu_{0}\left[\begin{array}{ccc}
\mu_{r} & 0 & 0 \\
0 & \mu_{r} & 0 \\
0 & 0 & \mu_{z}
\end{array}\right],
$$

where

$$
\mu_{r}=\mu+\kappa=\mu_{r}^{\prime}-j \mu_{r}^{\prime \prime}=1+\frac{H_{0 r}+\hat{w}+j \alpha \hat{w}}{H_{0 r}^{2}-\hat{w}^{2}+2 j \alpha H_{0 r} \hat{w}},
$$

where $H_{0 r}=H_{0} / M_{\mathrm{S}}, \hat{w}=\hat{f} / f_{m}, f_{m}=\gamma M_{\mathrm{S}}, \gamma=35.217$ $\mathrm{MHz} /(\mathrm{kA} / \mathrm{m}), \alpha$ is the Gilbert damping factor, and $\hat{f}$ is the complex frequency of FO. The Gilbert damping factor $\alpha$ is often replaced with the ferromagnetic linewidth $\Delta H=$ $2 \alpha H_{0}$.

For the lossless case

$$
\mu_{r}=1+\frac{1}{H_{0 r}-w},
$$

where $w$ is the real part of $\hat{w}$.

\section{Appendix B}

The resonance condition (characteristic equation) for the $\mathrm{TE}_{n m p}$ modes (subscripts $n, m, p$ refer to $\theta, \varphi, \gamma$ spherical coordinates, respectively) of FO of an isotropic sphere having diameter $d$ permittivity $\varepsilon_{r}$, and permeability $\mu_{r}$ situated in a dielectric medium of permittivity $\varepsilon_{d}$ has the following form:

$$
\begin{gathered}
\left\{n J_{n+\frac{1}{2}}(k d / 2)-k J_{n-\frac{1}{2}}(k d / 2)\right\} \\
H_{n+\frac{1}{2}}^{(2)}\left(k_{0} d / 2\right)-\mu_{r}
\end{gathered}
$$




$$
\begin{aligned}
& \left\{n H_{n+\frac{1}{2}}^{(2)}\left(k_{0} d / 2\right)-k_{0} H_{n-\frac{1}{2}}^{(2)}\left(k_{0} d / 2\right)\right\} \\
& J_{n+\frac{1}{2}}(k d / 2)=0
\end{aligned}
$$

where $k=\hat{\omega} / c\left(\varepsilon_{r} \mu_{r}\right)^{0.5}, k_{0}=\hat{\omega} / c\left(\varepsilon_{d}\right)^{0.5}, \varepsilon_{r}$ is the relative complex permittivity of the sphere, $\mu_{r}$ is the relative complex permeability of the sphere, $\varepsilon_{d}$ is relative complex permittivity of medium surrounding the sphere, $n, p$ are elevation and radial mode indices, respectively, $c$ is the speed of an EM wave in vacuum, and $\mathrm{J}(\mathrm{H})$ symbols denote Bessel (Hankel) functions.

Similarly, the characteristic equation for the $\mathrm{TM}_{n m p}$ modes can be formulated in the following form:

$$
\begin{aligned}
& \varepsilon_{d}\left\{n J_{n+\frac{1}{2}}(k d / 2)-k J_{n-\frac{1}{2}}(k d / 2)\right\} \\
& H_{n+\frac{1}{2}}^{(2)}\left(k_{0} d / 2\right)-\varepsilon_{r} \\
&\left\{n H_{n+\frac{1}{2}}^{(2)}\left(k_{0} d / 2\right)-k_{0} H_{n-\frac{1}{2}}^{(2)}\left(k_{0} d / 2\right)\right\} \\
& J_{n+\frac{1}{2}}(k d / 2)=0 .
\end{aligned}
$$

The radial mode index, $p$, denotes subsequent roots of (B.1) or (B.2). For isotropic media, modes are $(2 n+1)$-fold degenerated with respect to the index $m$, so one can assume $m=0$ without any change to the resonance frequencies. Frequencies of $\mathrm{FO}$ of $\mathrm{TE}_{n m p}$ modes can be found by solving (B.1) and for $\mathrm{TM}_{n m p}$ modes by solving (B.2) for given sample parameters.

\section{References}

1. G. Mie, Annalen der Physik 25, 377 (1908)

2. G. Shah, Numerical methods for Mie theory of scattering by a sphere. Kodalkenel Obs. Bull. Ser. A 2, 42-63 (1977)

3. Matlab program with Mie theory implemented. Available: https://scattport.org/index.php/programs-menu/ mie-type-codes-menu/111-mie-matlab-maetzler

4. C. Matzler, MATLAB functions for Mie scattering and absorption. Institute of Applied Physics, Bern, Switzerland, Research Report No. 2002-08 (2002)

5. C.F. Bohren, D.R. Huffman, Absorption and Scattering of Light by Small Particles (Wiley, New York, 1983)

6. S. Maier, Plasmonics: Fundamentals and Applications (Springer Science + Business Media LLC, New York, 2007)

7. J. Mevel, Etude de la structure detaillee des courbes de diffusion des ondes electromagnetiques par les spheres dielectriques. J. Phys. Radium 19, 630-636 (1958)

8. P. Chylek, Partial-wave resonances and the ripple structure in the Mie normalized extinction cross section. J. Opt. Soc. Am. 66, 285-287 (1976)
9. R. Fuchs, K.L. Kliewar, Optical modes of vibration in an ionic crystal sphere. J. Opt. Soc. Am. 58, 319-330 (1968)

10. P. Chylek, J.T. Kiehl, K.M. Ko, Optical levitation and partial wave resonances. Phys. Rev. A 18, 2229-2233 (1978)

11. H.J. Von Metz, H.K. Dettmar, Zur Berechung der Miescen Streukoeffizienten fur reele Brechungsindizes. Kolloid-Zeitschrift und Zeitschrift fur Polymere 192, 112 (1963)

12. A.D. Papatsoris, P.A. Watson, A rigorous explanation for the resonances observed in the scattering from spherical ice particles. IEEE Trans. Antennas Propag. 42, 1350-1354 (1994)

13. S.C. Hill, P. Chylek, Comment on "A rigorous explanation for the resonances observed in the scattering from spherical ice particles". IEEE Trans. Antennas Propag. 44, 1052-1055 (1996)

14. A. Julien, P. Guillon, Electromagnetic analysis of spherical dielectric shielded resonators. IEEE Trans. Microw. Theory Tech. 34(6), 723-729 (1986)

15. J. Krupka, M.E. Tobar, J.G. Hartnett, D. Cros, J. Le Floch, Extremely high-Q factor dielectric resonators for millimeter-wave applications. IEEE Trans. Microw. Theory Tech. 53(2), 702-712 (2005)

16. J. Krupka, B. Salski, P. Kopyt, W. Gwarek, Electrodynamic study of YIG filters and resonators. Sci. Rep. 6 (2016)

17. J. Krupka, P. Aleshkevych, B. Salski, P. Kopyt, A. Pacewicz, Ferromagnetic resonance revisedelectrodynamic approach. Sci. Rep. 6 (2017)

18. J. Krupka, P. Aleshkevych, B. Salski, P. Kopyt, J. Hartnett, Magnetic and electric solid-state plasmon spherical resonators. Plasmonics 14, 945-950 (2019)

19. J. Krupka, A. Pacewicz, B. Salski, P. Kopyt, J. Bourhill, M. Goryachev, M. Tobar, Resonances in large ferrimagnetic YIG samples - electrodynamic analysis. J. Magn. Magn. Mater. 521, 167536 (2021)

20. P.C. Fletcher, R.O. Bell, Ferrimagnetic resonance modes in spheres. J. Appl. Phys. 30, 687-698 (1959)

21. R.A. Hurd, The magnetic fields of a ferrite ellipsoid. Can. J. Phys. 36, 1073-1083 (1958)

22. J.E. Marcereau, Ferromagnetic resonance $\mathrm{g}$ factor to order $\left(k R_{0}\right)^{2}$. J. Appl. Phys. 30, 184-185 (1959)

23. J. Krupka, A. Pacewicz, B. Salski, P. Kopyt, J. Bourhill, M. Goryachev, M. Tobar, Electrodynamic improvements to the theory of magnetostatic modes in ferrimagnetic spheres and their applications to saturation magnetization measurements. J. Magn. Magn. Mater. 487, 165331 (2019)

24. D. Polder, Physica 15(40), 253 (1949)

25. A.G. Gurevich, Ferrites at Microwave Frequencies (Consultants Bureau Enterprises Inc., New York, 1963) 